\section{ASS kann Darmkrebs verhindern}

\section{Mehrere Studien haben gezeigt, dass ASS und COX-2-Hemmer Rezidive von Kolonadenomen verhindern können. Es war jedoch bislang nicht möglich, eine Senkung des Darmkrebsrisikos durch diese Medikamente nachzuweisen.}

- Rothwell et al. werteten die Daten von vier placebokontrollierten Studien über ASS in der primären und sekundären kardiovaskulären Prävention und einer Studie mit verschiedenen Aspirindosierungen aus. Die individuellen Patientendaten wurden gepoolt und der Effekt von ASS auf das Risiko für Darmkrebs nach 20 Jahren (Studiendauer und Nachbeobachtung) ausgewertet. Die eingesetzten Aspirindosierungen lagen bei 75-300 mg täglich.

Die Einnahme von Aspirin reduzierte das 20-Jahres-Risiko für Darmkrebs signifikant (Hazard Ratio HR $0,76 ; \mathrm{p}=0,02)$. Die Mortalität wurde ebenfalls signifikant gesenkt (HR 0,65; $\mathrm{p}=0,005)$. In der Gesamtgruppe war das Rektumkarzinomrisiko nicht signifikant gesenkt, hingegen gab es eine deutliche Senkung auch des Rektumkarzinomrisikos in der Gruppe, die fünf Jahre oder länger ASS eingenommen

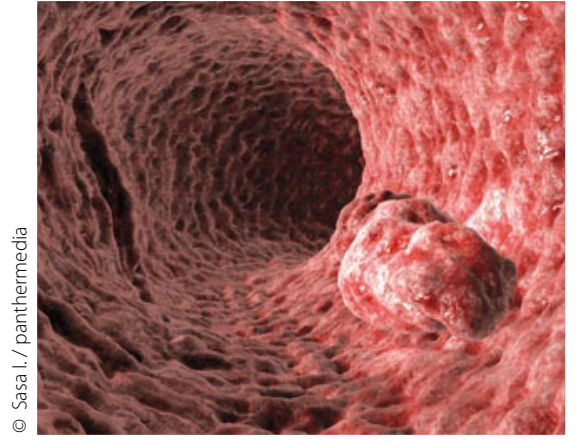

ASS schützt auch in niedriger Dosierung vor dem Darmkrebs.

hatte. Die absolute Risikoreduktion, innerhalb von 20 Jahren nach mindestens 5-jähriger Aspirin-Einnahme an einem kolorektalen Karzinom zu versterben, betrug $1,76 \%(\mathrm{p}=0,001)$.

- P. M. Rothwell et al.

Long-term effect of aspirin on colorectal cancer incidence and mortality: 20-year follow-up of five randomised trials. Lancet. 376 (2010) $1741-1750$
Kommentar

Diese Studie belegt eindrücklich, dass die dauerhafte Aspirin-Einnahme in einer kardiovaskulären Dosierung (75-300 mg täglich) zu einer Senkung des Darmkrebsrisikos und auch zu einer beindruckenden Senkung der Mortalität an Darmkrebs führt.

Bedeutet dies nun, dass alle Risikopersonen (z.B. Angehörige von Darmkrebspatienten oder Patienten mit kolorektalen Adenomen in der Vorgeschichte) prophylaktisch mit Aspirin behandelt werden sollen? Dies ist sicher nicht der Fall. Zum einen müssen die Nebenwirkungen, insbesondere das gastrointestinale Blutungsrisiko, einer Aspirin-Dauertherapie dem Nutzen gegenüber gestellt werden. Zweitens muss der Schwerpunkt unverändert auf der Vorsorgekoloskopie und den Überwachungskoloskopien der Risikopatienten liegen. Durch diese Maßnahmen kann das Darmkrebsrisiko sicher deutlich stärker als durch eine ASS-Medikation gesenkt werden, auch wenn hierzu wahrscheinlich nie randomisierte Studien durchgeführt werden.

Prof. Dr. MANFred Gross

\title{
Immer weniger Herzinfarkte
}

In den letzten 20 Jahren ist die Inzidenz des Herzinfarktes in den Industrieländern rückläufig. Woran das liegt, haben jetzt Wissenschaftler des University College London untersucht.

— In der sog. Whitehall-Studie wurden seit 1985 mehr als 103000 Beschäftigte des öffentlichen Dienstes untersucht. $\mathrm{Zu}$ Beginn waren die Teilnehmer 35 bis 55 Jahre alt. Insgesamt erlitten 256 von Ihnen einen Herzinfarkt (208 Männer und 48 Frauen).

Die periodische Auswertung der Daten ergab, dass die Herzinfarktrate in 20 Jahren jährlich um 6,5\% gesunken ist. Mehr als die Hälfte des Rückgangs konnten durch Veränderung von fünf Risikofaktoren erklärt werden. Im Vergleich zu 1985 rauchten 6\% (2-14\%) weniger, ihr systolischer Blutdruck war niedriger (13\%, 7-24\%), das „gute“ HDL-Cholesterin höher $(17 \%, 10-32 \%)$ und das LDL-Cholesterin niedriger. Die Probanden aßen mehr Obst und Gemüse. Negativ wirkte sich der Anstieg des BMI aus. Er reduzierte den Herzinfarkt-Rückgang um 11\% (5-23\%)

\footnotetext{
- Hardoon, Sarah L et al.

Rising adiposity curbing decline in the incidence of myocardial infarction: 20-year follow-up of British men and women in the Whitehall II cohort . Published in advance access on 8 June 2011 in Eur. Heart J.; doi:10.1093/eurheartj/her 142
}

\section{Kommenta}

Die Studie zeigt, dass die Aufklärungsarbeit über die Risikofaktoren des Myokardinfarkts doch Früchte trägt. Es sind überwiegend die Folgen eines veränderten Lebensstils, die sich in der White-Hall-Studie niederschlagen. Hätte im Untersuchungszeitraum nicht der Anteil der stark Übergewichtigen deutlich zugenommen, wäre der positive Trend noch wesentlich deutlicher ausgefallen. Was die Studie nicht zeigt, sind die positiven Effekte einer verbesserten Früherkennung und Therapie. 